\title{
SHORT- TO MEDIUM-RANGE OCEAN FORECASTS: DELIVERY AND OBSERVATIONAL REQUIREMENTS
}

\author{
Gary B. Brassington ${ }^{(1)}$, Adrian Hines ${ }^{(2)}$, Eric Dombrowsky ${ }^{(3)}$, Shiro Ishizaki ${ }^{(4)}$ Frank Bub $^{(5)}$, Mark \\ Ignaszewski $^{(6)}$
${ }^{(1)}$ Centre for Australian Weather and Climate Research, A partnership of the Bureau of Meteorology and CSIRO, Melbourne 3001, Victoria, Australia, Email: g.brassington@bom.gov.au
${ }^{(2)}$ Met Office, FitzRoy Road, Exeter, Devon EX1 3PB, United Kingdom, Email: adrian.hines@metoffice.gov.uk
${ }^{(3)}$ Mercator Océan, Parc Technologique du Canal, 8-10 rue Hermès, 31520 Ramonville St Agne, France, Email: Eric.Dombrowsky@mercator-ocean.fr Tokyo, Japan, Email: $\underline{\text { s_ishizaki@met.kishou.go.jp }}$
${ }^{(5)}$ Naval Oceanographic Office, Stennis Space Center, MS 39529, USA, Email: Frank.Bub@navy.mil
${ }^{(6)}$ Fleet Numerical Meteorology and Oceanography Center, 7 Grace Hopper Avenue, Monterey, CA 93943, USA,

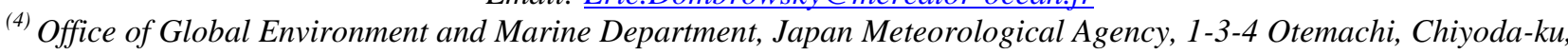
Email: Mark.Ignaszewski@navy.mil

\section{SYNOPSIS}

The Global Ocean Data Assimilation Experiment (GODAE) proposed in OceanObs'99 has proved so successful at demonstrating the practical utility of ocean forecasting that many research systems have been implemented as "operational" systems. GODAE has demonstrated that the global ocean observing system over the past decade can constrain an eddy-resolving ocean model to achieve skilful nowcasts and forecasts. In turn, these services are supporting a rapidly growing user community and a wide range of applications.

Mesoscale ocean circulation is a frontier science. Ocean eddies have been found to be ubiquitous over the entire ocean, accounting for more than $50 \%$ of the dynamic height variability. Evidence has recently emerged that geostrophic turbulence can also be systematic and contribute to the mean circulation. Further, model simulations indicate that eddies can extend into the abyssal depths supporting a complex range of stratified and un-stratified interactions that are difficult to substantiate with the present observing system. Intensive observational campaigns are required to resolve hypotheses posed by both theory and models.

At present, there is no redundancy in the observing system for ocean forecasting, all observations available in real-time can be demonstrated to have a positive impact. However, the impact is determined by the quality, quantity, independence and the statistical covariance of the observations to the model ocean state and circulation variables. Satellite altimetry has a dominant impact on global performance metrics due to its coverage and statistical covariance with the subsurface ocean state. Satellite altimetry is therefore the highest priority platform for ocean forecasting. Improving the real-time coverage would improve both performance and robustness. Satellite SST (Sea Surface Temperature) has a high impact on the upper ocean state estimation but less direct impact at depth. Satellite SST provides an important constraint to a multi-variate analysis with altimetry. Maintenance of present multisensor, multi-platform satellite SST is a priority. The direct impact of in situ observations is high local to the observation but the overall impact is limited by the short time/space covariances of the ocean model state and the coverage relative to that from remote sensing. The likelihood that in situ observations are available local to a practical application is low at present coverage.

The optimality of ocean forecasting systems is an important property to keep in mind when specifying observational requirements. Less optimal systems by definition require more observations to achieve the same performance. Development in ocean forecasting is none the less constrained by the pace of scientific innovation and the computational resources available for this high-end application. Achieving the performance required by the user will require advances in all aspects of the system. It is however reasonable to claim that the present observing system under-samples the mesoscale variability.

Many important applications will be able to make progress through an incremental improvement to GOOS (Global Ocean Observing System) as it exists in 2009 such as fisheries management, marine park management and coupled weather forecasting. These applications either have more time to evaluate the forecasts, to improve decision making or have less sensitivity to the forecast errors. However, there are a set of applications for which there is an immediate need for a higher level of forecast performance (e.g., search and rescue, marine accident and emergency and defence). These applications are ad hoc and typically highly localised in time and in space. A practical alternative strategy to expanding GOOS by an order of magnitude to achieve this performance would be to locally and temporarily (over the period of an event) enhance the observing 
system. Developing practical observation deployment strategies that maximise performance while minimising cost as well as developing national capabilities represent a significant challenge over the next decade. Some suggested components to a rapid and dense observing system are outlined.

There is a range of applications that will have high national and commercial value, which will be supported by the expansion of national observing networks predominantly focused within exclusive economic zones. Maximising progress across the range of applications will require both national and international planning and coordination between GOOS and these national and commercial efforts.

Robustness and consistency of the forecast performance is essential to the delivery of quality services and achieving the maximum impact to applications. At present, the performance is acutely sensitive to changes to the observing system. Improving the integrity of the observing system and related downstream product delivery infrastructure will improve the robustness of existing ocean forecast systems. The global observing system, open data access policies, real-time reporting have all played roles in achieving rapid innovation and capacity building. Maintaining these ingredients and making strategic and targeted investments over the next decade will support both the next generation of systems as well as the current and future development of systems within the Asia, Africa and South America.

\section{INTRODUCTION}

The Global Ocean Data Assimilation Experiment (GODAE) proposed in OceanObs'99 [1 and 2] has seen the emergence of sophisticated "operational" ocean prediction systems [3]. The term operational, refers to the robust and routine delivery of products and downstream services maintaining a consistent performance. A critical assessment of each component of the system, particularly the ocean observing system, does not satisfy this definition. Realistically, ocean forecasting is still in a pre-operational phase.

Forecast systems are composed of an assimilation step, which combines observations with a model background field, an initialisation step and, the final step, a forward model integration. The specific methods used in each step have an important impact on the overall performance of the system. This has important implications for assimilate satellite altimetry, satellite SST (Sea Surface Temperature) and in situ observations available in real-time [3]. The ocean state and circulation remains under observed for the mesoscale variability such that there is essentially no redundancy within the observing system. However, the impact of an individual observation is limited by the temporal and spatial covariances, so that coverage in time and space underpins performance. Current systems are sensitive to the consistency of the real-time observing system. For example the absence of one satellite altimeter in real-time leads to immediate deteriorations in performance that take several forecast cycles to recover once the data stream has been restored.

We begin by grouping applications that lend themselves to a common set of service requirements and examine a specific case study to outline the impact ocean forecasting is having on real-time events. We then discuss new discoveries in geostrophic turbulence and the implications for models and observations. We then briefly outline the status of current operational ocean forecasting systems and then outline the observational requirements for these systems. We draw particular attention to the set of applications that will not be adequately served by incremental improvements to GOOS where novel approaches such as adaptive sampling would yield higher performance at a more modest cost. Finally, we outline the role this community will need to play in justifying the observational requirements. The synopsis and conclusion serve to summarise this material.

\section{APPLICATIONS GROUPED BY THEIR SERVICE REQUIREMENTS}

There is no definitive way to group applications of ocean services and many groupings are possible. However, for the present purpose we choose to group applications that lend themselves to a translation into observational requirements. We acknowledge at the outset that the grouping presented is coarse grained for which there will be exceptions and that the complete observational requirements will need to be obtained through the collective assessment of individual applications. The significance and frequency of each application area will vary from nation to nation with downstream impacts with respect to the support for elements of GOOS (Global Ocean Observing System) and national observing networks. Each application group identified has been tabulated to outline some basic properties and in particular a gross assessment of their observational requirements. 


\subsection{Ad hoc time and space}

\begin{tabular}{|l|l|}
\hline Service type & Public good / Defence \\
\hline $\begin{array}{l}\text { Specific } \\
\text { applications }\end{array}$ & $\begin{array}{l}\text { Search and Rescue (SAR), Marine } \\
\text { Accident and Emergency Services } \\
\text { (MAES), criminal investigations, } \\
\text { insurance, TC's and storms, coastal surge, } \\
\text { defence and national security operations }\end{array}$ \\
\hline $\begin{array}{l}\text { Service } \\
\text { providers }\end{array}$ & National operators / Defence \\
\hline $\begin{array}{l}\text { Service } \\
\text { requirements }\end{array}$ & $\begin{array}{l}\text { Ad hoc location and time, high precision } \\
\text { nowcasts; skilful forecasts of surface state } \\
\text { and circulation }\end{array}$ \\
\hline $\begin{array}{l}\text { Observational } \\
\text { requirements }\end{array}$ & $\begin{array}{l}\text { Rapid, intensive sampling; sustained for } \\
\text { the period of the incident; not satisfied by } \\
\text { GOOS-2009 } \\
\text { Unlikely to be satisfied by +10-20\% GOOS }\end{array}$ \\
\hline References & [4,5,6,7] \\
\hline
\end{tabular}

Table 2.1 A summary of the properties of ad hoc time and space applications

\subsection{Planning and management}

\begin{tabular}{|l|l|}
\hline Service type & Public good / industrial \\
\hline $\begin{array}{l}\text { Specific } \\
\text { applications }\end{array}$ & $\begin{array}{l}\text { Coral bleaching, fisheries management, bi- } \\
\text { catch, ship-routing, offshore ship } \\
\text { operations }\end{array}$ \\
\hline $\begin{array}{l}\text { Service } \\
\text { providers }\end{array}$ & National operators / Third party providers \\
\hline $\begin{array}{l}\text { Service } \\
\text { requirements }\end{array}$ & $\begin{array}{l}\text { Fixed location, routine services, seamless } \\
\text { prediction required short- medium, } \\
\text { seasonal- }\end{array}$ \\
\hline $\begin{array}{l}\text { Observational } \\
\text { requirements }\end{array}$ & $\begin{array}{l}\text { Homogeneous basin / marginal sea, } \\
\text { Sustained, long-term observations, GOOS- } \\
2009 \text { is impacting this application } \\
\text { Likely to be satisfied by +10-20\% GOOS }\end{array}$ \\
\hline References & {$[4]$} \\
\hline
\end{tabular}

Table 2.2 A summary of the properties of planning and management applications

\subsection{Engineering/Industrial/Businesses}

\begin{tabular}{|l|l|}
\hline Service type & Public good / industrial \\
\hline $\begin{array}{l}\text { Specific } \\
\text { applications }\end{array}$ & $\begin{array}{l}\text { Offshore platforms, renewable energy sites, } \\
\text { eco -tourism, desalination discharge }\end{array}$ \\
\hline $\begin{array}{l}\text { Service } \\
\text { providers }\end{array}$ & National operators / Third party providers \\
\hline $\begin{array}{l}\text { Service } \\
\text { requirements }\end{array}$ & $\begin{array}{l}\text { Fixed location, routine services, seamless } \\
\text { prediction required short- medium-, value- } \\
\text { added to GOOS-derived services, National } \\
\text { and multi-national drivers }\end{array}$ \\
\hline $\begin{array}{l}\text { Observational } \\
\text { requirements }\end{array}$ & $\begin{array}{l}\text { Not satisfied by GOOS-2009, observations } \\
\text { target value-adding to GOOS, capacity to } \\
\text { deploy commercial instrumentation } \\
\text { Requirement to encourage integration } \\
\text { through common data standards and open } \\
\text { access to national operators and long-term } \\
\text { archives }\end{array}$ \\
\hline References & [4] \\
\hline
\end{tabular}

Table 2.3 A summary of the properties of engineering and industrial applications

\subsection{Global and continuous}

\begin{tabular}{|l|l|}
\hline Service type & Public good \\
\hline $\begin{array}{l}\text { Specific } \\
\text { applications }\end{array}$ & $\begin{array}{l}\text { Weather, waves, bio-geo-chem, carbon } \\
\text { accounting }\end{array}$ \\
\hline $\begin{array}{l}\text { Service } \\
\text { providers }\end{array}$ & National operators \\
\hline $\begin{array}{l}\text { Service } \\
\text { requirements }\end{array}$ & $\begin{array}{l}\text { Global, routine services, seamless } \\
\text { prediction required short- medium, } \\
\text { seasonal- }\end{array}$ \\
\hline $\begin{array}{l}\text { Observational } \\
\text { requirements }\end{array}$ & $\begin{array}{l}\text { Homogeneous basin / marginal sea, } \\
\text { Sustained, long-term } \\
\text { Progress can be made with GOOS-2009 } \\
\text { May be satisfied by incremental increases } \\
\text { to GOOS }\end{array}$ \\
\hline References & [7,8] \\
\hline
\end{tabular}

Table 2.4 A summary of the properties of global and continuous applications 


\subsection{Coastal / shelf}

\begin{tabular}{|l|l|}
\hline Service type & Public good / Industry \\
\hline $\begin{array}{l}\text { Specific } \\
\text { applications }\end{array}$ & $\begin{array}{l}\text { Port management, bilge discharge, coastal } \\
\text { weather, coastal surge and management }\end{array}$ \\
\hline $\begin{array}{l}\text { Service } \\
\text { providers }\end{array}$ & National operators / third party providers \\
\hline $\begin{array}{l}\text { Service } \\
\text { requirements }\end{array}$ & $\begin{array}{l}\text { Global, routine services, seamless } \\
\text { prediction required short- medium, } \\
\text { seasonal-, National drivers }\end{array}$ \\
\hline $\begin{array}{l}\text { Observational } \\
\text { requirements }\end{array}$ & $\begin{array}{l}\text { Coastal observations integrated with } \\
\text { GOOS, Sustained, long-term observations, } \\
\text { GOOS-2009 is impacting this application } \\
\text { Likely to be satisfied by +10-20\% GOOS } \\
\text { Requirement to encourage integration } \\
\text { through common data standards and open } \\
\text { access to national operators and long-term } \\
\text { archives }\end{array}$ \\
\hline
\end{tabular}

Table 2.5 A summary of the properties of coastal/shelf applications

2.6 Public good / public warnings

\begin{tabular}{|l|l|}
\hline Service type & Public good \\
\hline $\begin{array}{l}\text { Specific } \\
\text { applications }\end{array}$ & $\begin{array}{l}\text { Research, recreational fishing, diving, } \\
\text { swimming, yacht racing, adventurers }\end{array}$ \\
\hline $\begin{array}{l}\text { Service } \\
\text { providers }\end{array}$ & National operators \\
\hline $\begin{array}{l}\text { Service } \\
\text { requirements }\end{array}$ & $\begin{array}{l}\text { Global, routine services, moderate } \\
\text { accuracy/skilful nowcast/forecasts of } \\
\text { surface state and circulation National } \\
\text { drivers }\end{array}$ \\
\hline $\begin{array}{l}\text { Observational } \\
\text { requirements }\end{array}$ & $\begin{array}{l}\text { Coastal observations integrated with } \\
\text { GOOS, Sustained, long-term observations, } \\
\text { GOOS-2009 is impacting this application } \\
\text { Likely to be satisfied by +10-20\% GOOS } \\
\text { Requirement to encourage integration } \\
\text { through common data standards and open } \\
\text { access to national operators and long-term } \\
\text { archives }\end{array}$ \\
\hline
\end{tabular}

Table 2.6 A summary of the properties of pubic good applications

\section{WORKED EXAMPLE}

Making progress in the specification of ocean forecast service requirements will require the consolidation of many "worked examples" together with engagement with relevant user communities to provide detailed assessment of the impact of these services. Obtaining the quality of information that is necessary to make a robust case for improved services and observing system requirements is a complex task. Achieving this level of engagement with users has been modest and slow to develop. For many applications such as accidents and emergencies, the user is an operator whose primary concern is the immediate incident. Although information gathering in real-time is the best means of obtaining the quality information, it is a distraction to the operator. As the quality of the ocean forecasts improves and increasingly reliable for operational planning decisions the relationship will improve over the next decade.

Organisations such as MyOcean, US-IOOS (United States-Integrated Ocean Observing System) and the JCOMM (Joint Technical Commission for Oceanography and Marine Meteorology) Expert Team for Operational Ocean Forecast Systems (ET-OOFS) will provide national and international coordination to the information gathering and develop service requirements. Many examples will emerge in the future, for which high quality information can be gathered. The following was a real-time example at the time of the conference

\section{Montara well oil spill}

On the morning of Friday 21 August 2009, the West Atlas mobile drilling unit was engaged in well operations at the Montara Well in the Timor Sea. For reasons still unknown, the drilling rig had an uncontrolled release of oil and gas to the environment of the Timor Sea. Fortunately, there were no casualties, and the rig was abandoned. The oil spill response was expected to be protracted and logistically demanding. Continuous monitoring, plume trajectory predictions and dispersant operations were required for around eight weeks. The oil spill officially ceased on 3rd November 2010 .

The slick remained offshore away from coastlines and coral reefs throughout the event. The Australian Maritime Safety Authority (AMSA) was tasked with coordinating the response to the oil spill and released regular updates to the public on the status. Figure 1a shows the oil plume discharging from the well in the first couple of days. Figure $1 \mathrm{~b}$ shows the aircraft deployed to drop dispersant on the oil spill and Fig. 1c shows ship-based containment equipment and extraction operations.

The Montara Well is located approximately $250 \mathrm{~km}$ north of the Kimberly's a region to the northwest of Australia's $250 \mathrm{~km}$ southeast of West Timor and approximately $150 \mathrm{~km}$ east of the Ashmore reef complex. This region of the Timor Sea has a depth of $200 \mathrm{~m}$, its circulation is influenced by tides, Indonesian Throughflow, and the formation of warm-core eddies. The atmospheric conditions during this period are characterised by weak winds / clear skies.

An ocean warm pool develops in this region, which leads to the onset of the Australian monsoon during the Austral summer. The region was frequently cloud free 

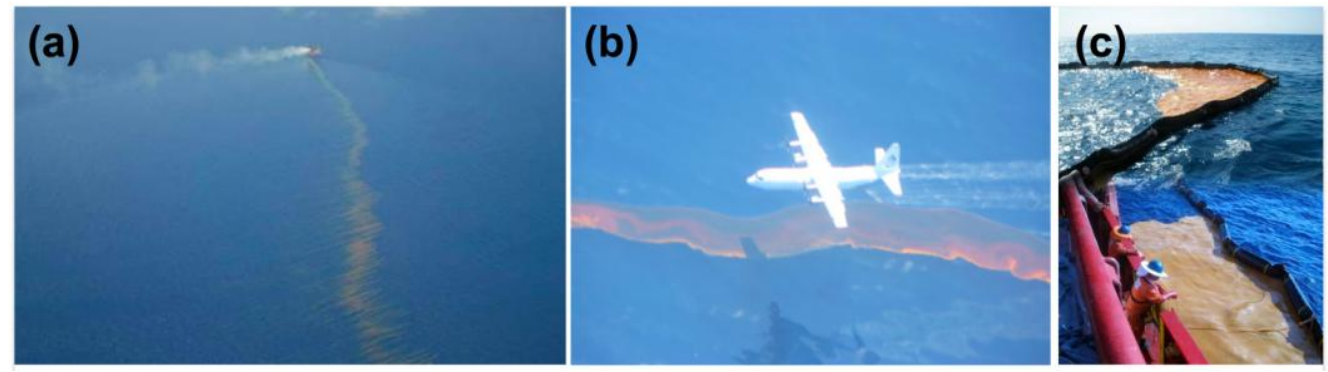

Figure 1: (a) Aerial reconnaissance photo of the Montara Oil Well and oil slick, (b) Aircraft operating to perform aerial drop of dispersant to the oil slick, (c) Ship-based operations deployed to contain and retrieve the oil slick. (Photos courtesy of the Australian Maritime Safety Authority).

permitting the oil spill to be observed by the MODIS (Moderate Resolution Imaging Spectroradiometer) instrument on Aqua ((EOS PM)) and Terra (EOS AM) satellites. The oil spill is visible via Channel $2(859 \mathrm{~nm})$ which offer $250 \mathrm{~m}$ resolution. Figure 2 shows a black and white insert for channel 2 with a background of the RGB color for geo-referencing.

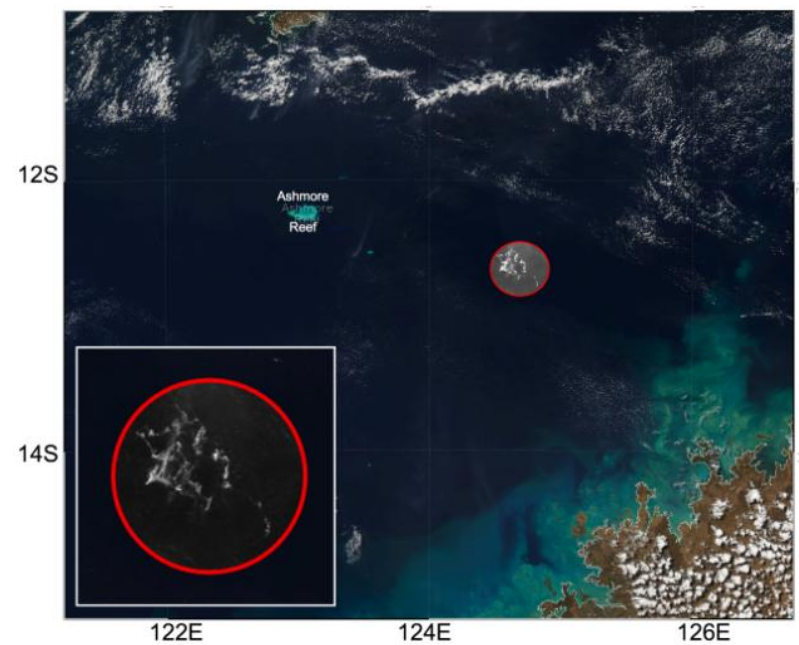

Figure 2: Image captured by MODIS (Aqua) on 23rd August 2009 at 05:15UTC. Data courtesy of NASA Ocean Biology Processing Group and the Australian Bureau of Meteorology (Leon Majewski). Insert is an expanded view of the suspected oil spill.

The atmospheric conditions in the weeks following the oil spill were weak local winds and clear skies. During this period, the oil slick was influenced by weak mesoscale circulation and meanders through tidal and inertial motion. Figure 3 shows a $48 \mathrm{hr}$ forecast depicting the seasonal easterly winds that developed to the north of Australia but did not directly impact the region of the oil spill. Over the subsequent month, the seasonal easterlies moved south influencing the Timor Sea although due to the El Nino conditions the trade winds were weaker than normal.

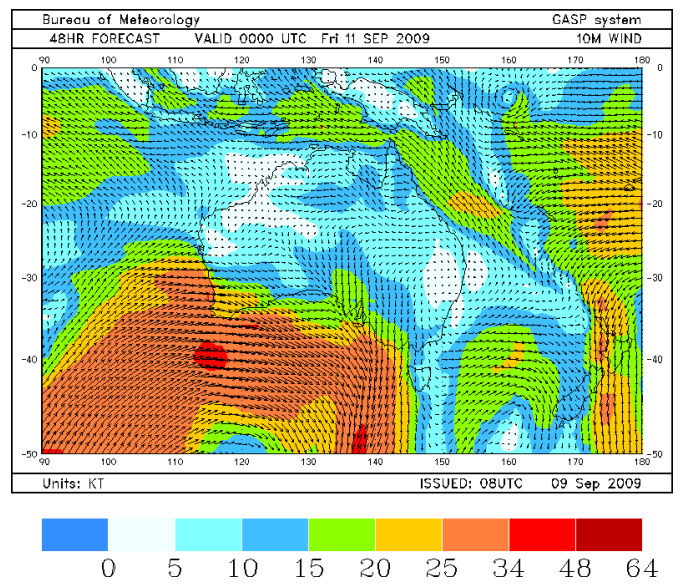

Figure 3: $48 \mathrm{hr}$ forecast of $10 \mathrm{~m}$ winds from the global weather prediction system [9] for the 11th September 2009. A band of easterlies has developed over northern Australia.

The Asia-Pacific Applied Science Associates (APASA) is a third party service provider that was contracted to provide AMSA with forecast services during the event. APASA specialise in oil spills and search and rescue type products. Recently they have configured their proprietary software OILMAP (Oil Spill Model and Response System) and SARMAP (Search and Rescue Model and Response System) with analysis and forecast products of BLUElink (Ocean forecasting Australia) OceanMAPSv1.0b [10]. This complements the existing products of the US Navy Coastal Ocean Model (NCOM) [11] and CSIRO (Commonwealth Scientific and Industrial Research Organisation) sea level analyses [12]. Figure 4 shows the estimated trajectory by APASA of the oil spill using the three available estimates of the mesoscale surface circulation combined with tide estimated by APASA and NOGAPS (Navy Operational Global Atmospheric Prediction System) winds. The trajectories overlay the clearest images from MODIS on the 30th August. The hind-analysis from GSLA (Gridded Sea Level Anomalies) does show good agreement with the main plume shown although it clearly misses the finer scale meandering. BLUElink 
OceanMAPSv1.0c and NCOM both appear to have a directional bias in the trajectories however, they provided to be the more reliable information for the forecast conditions.

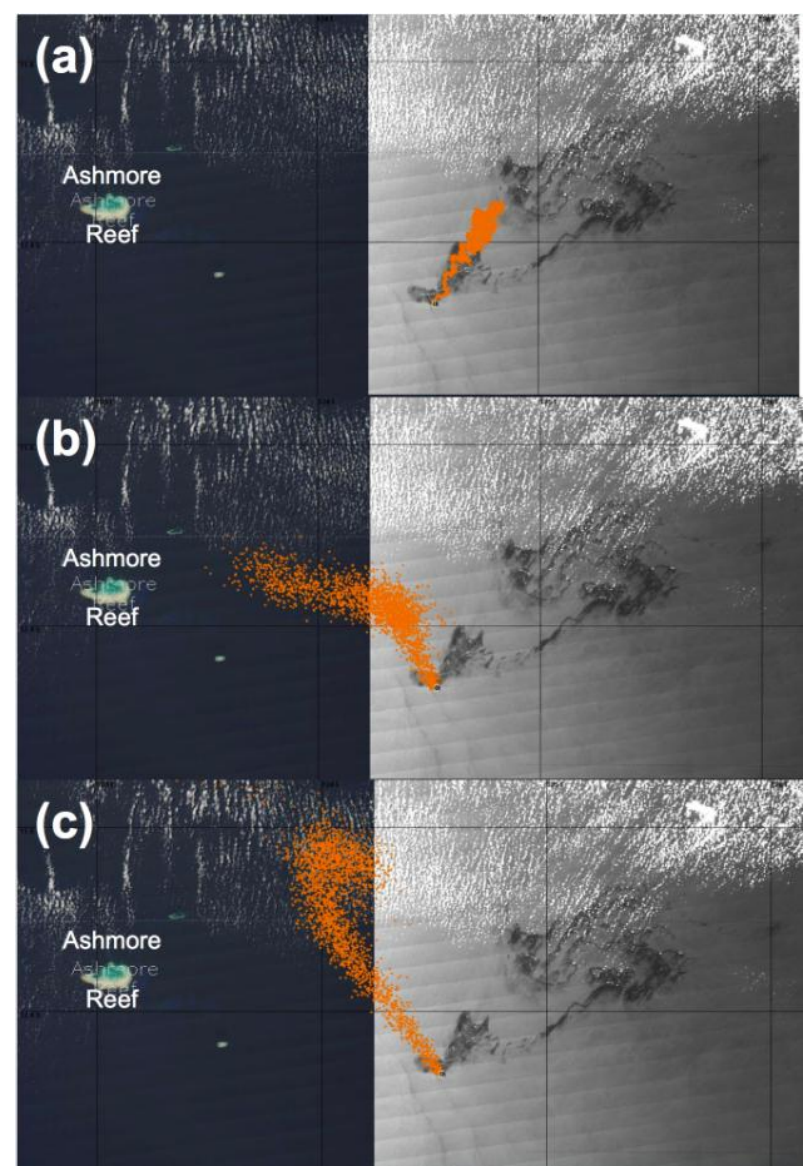

Figure 4: Estimated oil slick trajectories from OILMAP based on tides, NOGAPS winds and three ocean current products: (a) CSIRO GSLA, (b) NCOM and (c)

BLUElink OceanMAPSv1.0c [10]. The trajectories overlay the MODIS satellite image on the 3pm 30th August 2009

Further analysis of the BLUElink OceanMAPSv1.0c demonstrated objectively viewed as the most reliable forecast for the day shown in Fig. 4 and for the subsequent 40 days (Dr Brian King private communication). The apparent discrepancy shown in Fig. 4 can be explained if it is realised that the MODIS image represents the past behaviour of the ocean circulation and is not a useful comparison for the forecasts. The trajectories obtained from OceanMAPS ocean forecasts (with some consensus from NCOM) indicate a change in circulation that was validated by subsequent MODIS imagery as well as reconnaissance aircraft. The GSLA product represents a 4 to 5 day behind real-time hind analysis. The GSLA trajectories therefore show excellent agreement with the oil spill captured by the MODIS image shown. None the less, the close agreement shown by GSLA indicates that the mesoscale circulation is dominating the dispersion of the oil-seawater fluid in the Timor Sea. It also suggests that if the altimetry coverage currently available behind real-time were available in near real-time it would provide useful first order information for these applications, even in relatively shallow and marginal locations, as is the case here.

\section{GEOSTROPHIC TURBULENCE}

\subsection{DYNAMICS}

Mesoscale circulation (or geostrophic turbulence) is a frontier science with the properties of eddy-eddy unstratified and stratified interactions and their contribution to the mean circulation are active areas of research [13 and 14]. This field requires advances in theory, modelling and observations to make progress. There is a need for intensive observational campaigns that can resolve hypotheses posed by theory and models.

Figure 5 below shows the cross-correlation of daily average surface currents in the Tasman Sea from BLUElink OceanMAPSv1.0b [10] with a pattern of rotational flow. The local maxima (red) correspond to vortices in the circulation and medium correlations correspond to lines of maximum shear that partially align with the vectors of the rotational flow. The orientation and extent of coherent circulation is represented by the red/black ellipses, which correspond to anticyclonic/cyclonic, eddies. The Tasman Sea is a region occupied by the East Australian Current one of the worlds western boundary currents. Equivalent examples of geostrophic turbulence can be found within regions influenced by the Gulf Stream, Aghulas, Kuroshio and Brazil Currents. These regions are characterised by the largest dynamic height variability and thereby most energetic regions, although eddies have been found to be ubiquitous over the entire ocean accounting for more than $50 \%$ of the dynamic height variability [13]. The East Australian Current separates from the coast at $\sim 33 \mathrm{~S}$ and forms the Tasman Front separating warm/fresh Coral Sea water masses from cool/salty Tasman Sea water masses. Eddies are frequently formed from flow instabilities as either warm/fresh core anticyclonic eddies or cold/salty core cyclonic eddies. Figure 5 shows that the Tasman Sea tends to be dominated by a few large anticyclonic eddies $\mathrm{O}(200 \mathrm{~km})$ with a full spectrum of smaller eddies to the limit of the grid resolution of the model. The metric also identifies the shear flow associated with fronts, which frequently form new vortices. The major axis of the ellipse tends to be aligned with fronts and other like-signed eddies. The ocean circulation at any instant in time in the Tasman Sea is composed of a complex set of features, which continuously interact. 


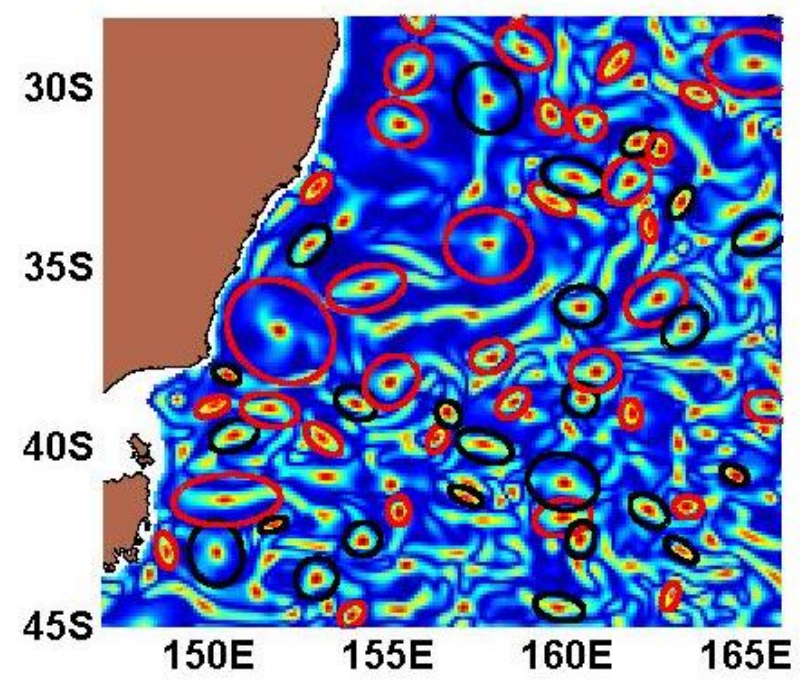

Figure 5: The cross-correlation of daily average surface currents in the Tasman Sea on the 18th Feb 2008 (from Oceanmapsv1.0c [10]) with a pattern of rotational flow. Each local maximum corresponds to an eddy. Overlaid are the approximate elliptical extent of each large anticyclonic eddy (red) and cyclonic eddy

(black). Courtesy of Mr. Nicolas Summons.

Extending the pattern matching technique to each layer of the ocean model we can identify the vertically coherent circulation of surface and sub-surface eddies as depicted by the model. To aid visualisation the coherent vortices are shown as iso-surfaces coloured red/blue to depict anti-cyclonic/cyclonic circulation (see Fig. 6). In the Tasman Sea, the vortices frequently extend to the full depth of the ocean up to $4000 \mathrm{~m}$ as shown here. The vertical axis of the simulated eddies is frequently not aligned with the gravitational vector through the work of eddy-mean flow, eddy-eddy and eddy-bathymetry interactions.

Since the dominant source of energy in this region comes from the warm/fresh (less dense) EAC, the majority of eddy formation occurs in the upper layer of the ocean. The upper layer of the ocean is occupied by both shallow and deep vortices, which shortens the spatial scales and increases the frequency of turbulent interactions. Coherent vortices in the deep model ocean are initially formed by large surface eddies that persist over a longer timescale. The spatial scales of separation are larger in the deep ocean indicating less frequent eddy-eddy turbulent interactions. The deep vortices are associated with a small geopotential anomaly compared with the surface and are a small contribution to the total surface height anomaly making them difficult to observe and verify. Therefore, it has not yet been determined whether these features are realistically represented by the model. Nonetheless, in the model they have important dynamical consequences for the upper layer turbulence.

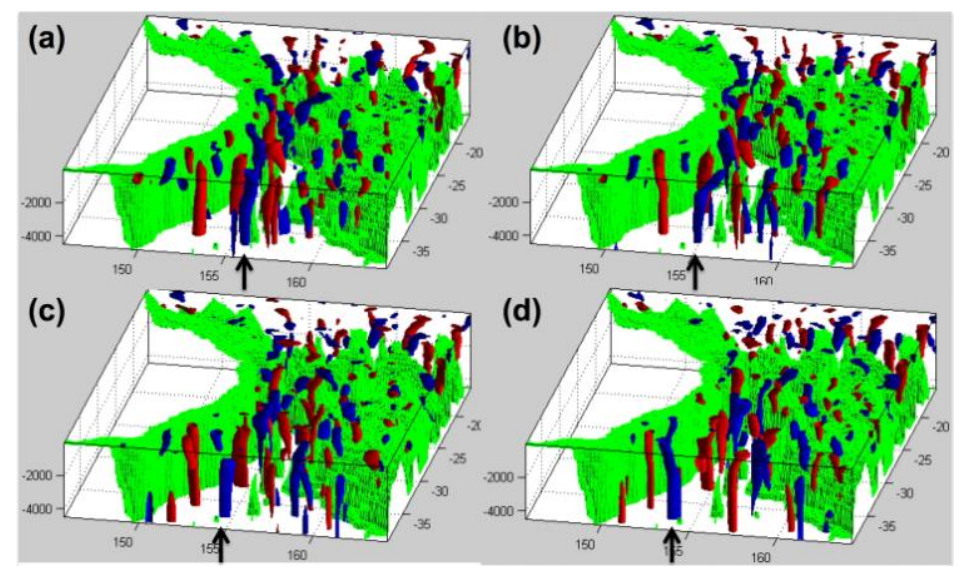

Figure 6: Iso-surfaces of the vertically coherent vortical circulation in the Tasman Sea derived from the daily average velocity from Oceanmapsv1.0c. Anticyclonic eddies (red) and cyclonic eddies (blue) are shown over a surface of the bathymetry. The arrow tracks the dynamic sheer and re-attachment of a deep cyclonic vortex

Figure 6a-d shows one example of an important interaction of a cyclonic eddy. In this case, the full depth cyclonic eddies shear at mid-depth to form a surface and deeper ocean vortex (see Fig. 6a and 6b) that separate. The deeper vortex persists on the timescale of weeks (see Fig. 6c) sufficient to begin interaction with another surface vortex. The two vortices proceed to undergo a stratified vortex merger, Fig. 6d.
The role of the deeper vortex on the upper ocean, in the model for this example, is therefore to retard surface interactions through the additional work involved in the sheer and can persist to attract another surface vortex and thereby impacting the water masses within the surface eddy. 


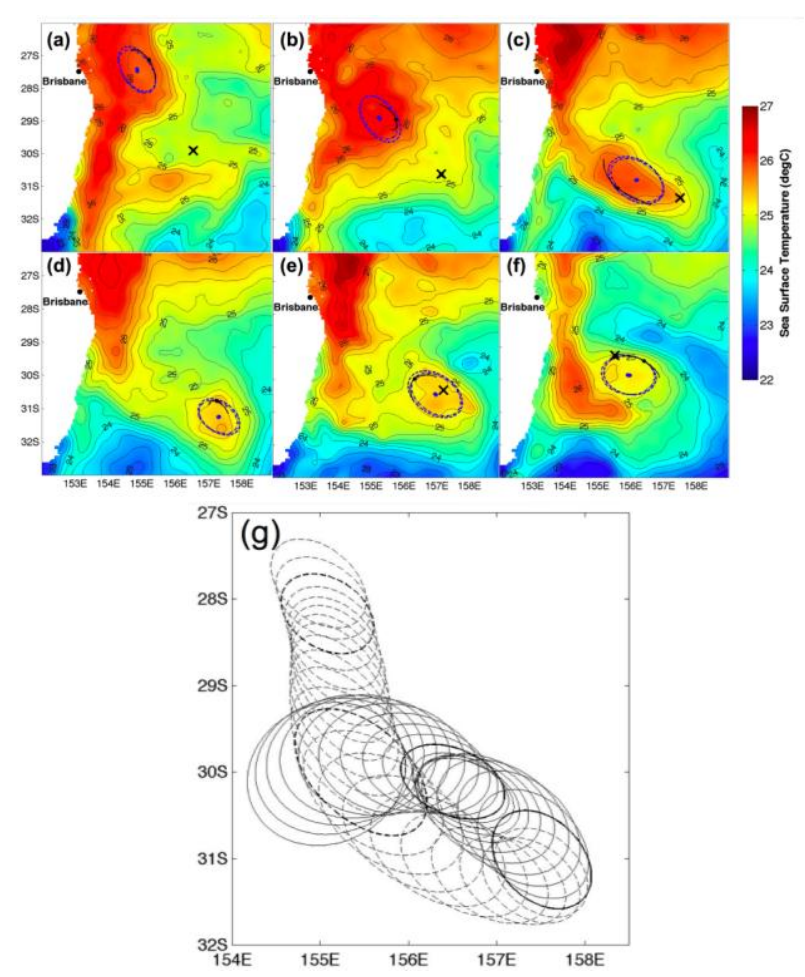

Figure 7: Estimated surface ellipse and buoy trajectories of WMO55916 and WMO55924 overlaid on UKMet OSTIA (Operational Sea Surface Temperature and Sea Ice Analysis) SST for (a) $26^{\text {th }} \mathrm{Feb},(b) 8^{\text {th }},(c)$ $17^{\text {th }}$, (d) $21^{\text {st }}$, (e) $27^{\text {th }}$ Mar and (f) $6^{\text {th }}$ Apr. (g) shows the

daily evolution of the ellipse estimated from the trajectory of WMO55916 [15].

A specific example of vortex generation in the Tasman Sea was observed by two surface drifting buoys that remained within the surface circulation for $\sim 60$ days completing nine (9) orbits of the surface vortex [15] and shown in Fig. 7. After the eddy separated from the coast the SST observations (see Fig. 7a-f) show that the eddies eastward propagation was arrested reaching a stationary point attributed to a stratified vortex merger [15]. Subsequent to the merger, the drifting buoys observe a surface convergence, depicted by the convergence of the material ellipse shown in Fig. $7 \mathrm{~g}$ derived from the drifting buoy trajectory resulting from the secondary circulation and vertical adjustment of the merged eddies. Mesoscale ocean circulation has a broad spectrum of temporal and spatial scales with new incites into organisation and vertical interactions. Constraining a mesoscale ocean analysis poses a significant challenge to the observing system.

\subsection{MODELLING}

The majority of the ocean modelling community has focused on either climate [16] or coastal oceanography as reflected by the majority of community models that are available. A modelling community specifically focused on resolving geostrophic turbulence is required to support further progress in global and basin scale ocean forecasting. Accurately evolving geostrophic turbulence over days to months leads to a different set of design decisions compared with evolving the ocean over years to decades. Fundamental choices such as Boussinesq vs non-Boussinesq models, Arakawa C-Grid vs B-Grid, so-pycnal/geopotential/sigma vertical coordinates, order of accuracy and conservation properties of numerical schemes are determined by the target application and frequently lead to inferior choices for secondary applications. The focus of parameterisations and closure schemes is also determined by the physical processes that are unresolved. Achieving scale independent closures are difficult to achieve and becomes a challenge when the shape of the energy spectrum changes due to key physical processes becoming partially resolved. Community ocean models that target the mesoscale ocean state and circulation will help to bring the ocean modelling and geostrophic turbulence communities closer together for the benefit of ocean science and ocean forecasting.

A limited number of studies have explored the impacts of resolution on ocean prediction [17 and 18]. These studies indicate that correctly resolving boundary current separation is dependent on resolving specific physical processes and bathymetric features but further grid refinements lead to only marginal improvement indicating an optimum can be specified in terms of computational efficiency. Model spinup studies provide guidance to the processes that can be resolved and detect the biases between observed and model represented ocean circulation. Ocean modelling however represents one component of an ocean prediction system. The total system includes the observing system, the quality control system, the analysis system, the initialisation that prevents the ocean-only performance being realised.

Present global and basin-scale operational prediction system resolutions are summarised in Tab. 1 in [3]. The horizontal resolution ranges from $1 / 4^{\circ}$ to $1 / 12^{\circ}$ (with NLOM (NRL (Naval Research Lab) Layered Ocean Model) $1 / 32^{\circ}$ ). Future plans from the majority of centres indicating the resolution over the next five years will increase to a range of $1 / 12^{\circ}$ to $1 / 25^{\circ}$. This resolution will produce improved skill over the continental shelf particularly for coastally trapped waves, coastal sea level and upwelling however these resolution remain coarse with respect to the resolution requirements of coastal models which target $1 \mathrm{~km}$ and finer. Nested, downscaled strategies will remain relevant over the next decade for coastal prediction.

Coupled short- and medium-range prediction will become mainstream over the next decade with developments being undertaken in the leading agencies to extend the weather prediction systems [19]. Current 
strategies are to adopt low-resolution ocean models or higher resolution mixed layer models for reduced computational cost. It is expected however that global ocean prediction systems will replace these simplified models to provide higher forecast skill of the air-sea interaction over the next decade [8]. Progress in coupled prediction will require improved boundary layer and flux parameterisations to improve the skill of the air-sea exchange over short- to medium-range timescales. Closure of errors in air-sea fluxes will increase the significance of covariance of background errors in observable surface properties of sea level anomaly, sea surface temperature, sea surface salinity and background errors in the atmosphere of air-temperature, specific humidity, wind, radiation, clouds and precipitation. Initial progress with high resolution global ocean prediction systems will be made through semicoupling of the global weather and wave prediction systems by sharing forecast surface properties in alternating forecast cycles. Full coupling will progress with regional models for which the tropical cyclone community are leading development of high-wind conditions [6].

\subsection{OCEAN DATA ASSIMILATION}

A critical part of an ocean forecasting system is to combine the sparse observations with a model-estimated state to derive a best estimate of the ocean state. The methodology commonly adopted in numerical weather prediction and now ocean prediction is referred to as data assimilation [20]. There are several variants of solvers and implementations however they all share a common fundamental equation based on a generalised least squares analysis for multiple state variables and multiple dimensions. A background or forecast estimate of the ocean state is provided by a model forecast which is interpolated to the observed position. The difference between the model and observed state variable, termed an innovation, is an estimate of the error of the background state from the true state. The confidence of that error is determined by the relative expected errors of the background and observation. Due to the continuity of the ocean state properties, it is expected that an error in one position will indicate errors in the surrounding region local to the observation. This information varies in space and time and is termed the background error covariance (BEC). Similarly the errors from multiple observations may also be correlated (e.g., along track errors) which can be captured in an observation error covariance (OEC).

There are several approaches that can be taken to estimate the BEC starting with simple assumptions of analytically defined Gaussian distributions, to the use of model ensembles. On the other hand, the OEC is frequently assumed to be uncorrelated. The primary trade-off taken in data assimilation relates to the optimality of the analysis and the computational cost where the model state space is $\mathrm{O}\left(10^{8}\right)$ and the observation state space is $\mathrm{O}\left(10^{6}\right)$.

The quality of the best estimate is directly related to the number of innovations that contribute to the least squares analysis at each point in state space. This is determined by the length scales of the BEC (i.e., the distance over which there is a covariance) and the density of observations. The BEC will contain multiple length scales related to the influence of different processes that contribute to the total error. Seasonal processes have timescales $\mathrm{O}$ (days to wks) and horizontal spatial scales $\mathrm{O}\left(10^{3}\right) \mathrm{m}$. Mesoscale processes have time scales $\mathrm{O}$ (days) and horizontal length scales $\mathrm{O}\left(10^{2}\right) \mathrm{m}$. Therefore, mesoscale analyses require an order of magnitude increase in the density of observations in space to achieve an equivalent analysis performance to that of seasonal.

As stated earlier the OEC is frequently assumed to be diagonal i.e., that the observation errors are independent and uncorrelated. This assumption is made in part for numerical convenience but also due to the absence of an accurate estimate of these statistical relationships. The analysis would be improved by including a more complete OEC but there are two challenges for practical implementation: (a) estimating the covariance relationships and (b) developing a compact representation for storage and numerical computation.

There are two additional aspects to practical data assimilation that explicitly limit the influence of an observation on the analysis. The first is localisation that imposes a length scale beyond which BEC are set to zero. This is introduced to avoid unrealistic far field covariances when represented by a small ensemble. The second is the inclusion of a representative error, which is, adds a penalty to the observation error and accounts for the portion of ocean variability that is unresolved by the model. Operational forecasting systems use conservative values that improve robustness but reduce the impact of observations.

\subsection{OBSERVABILITY AND PREDICTABILITY}

There are two first order problems in ocean prediction (a) to initialise the state and circulation of resolved ocean eddies with sufficient accuracy to achieve forecast skill over persistence and (b) initialise the depth and state of the mixed layer and sub-surface layers that may be entrained.

Ocean prediction systems have demonstrated that narrow-swath altimeters with repeat orbit periods of 9.9 and 35 days are able to constrain the mesoscale eddies with measurable, though modest, forecast skill, over persistence [21]. Present systems use so-called 3D assimilation schemes due to the practical constraints of computational cost. A compromise is made to use a time window to increase the number of observations to 
constrain the least squares analysis and restricting the observation window local to the time of the analysis. A larger time window is best suited to slow moving eddies while faster moving eddies will tend to be aliased or more diffuse. Increasing the number of narrow-swath altimeters or the introduction of wide-swath altimeters will minimise this compromise leading to greater nowcast accuracy of the global distribution of mesoscale eddies. Greater computational power will also permit more optimal 4D schemes to be adopted.

Constraining the mixed layer temperature is well served by the multi-sensor, multi-orbit coverage of sea surface temperature. Microwave sun-synchronous and IR Geostationary platforms provide higher coverage while IR polar orbiting provides higher accuracy in cloud free conditions. There are several known limitations to the use of SST with diurnal warming and skin effects and specific algorithms used to determine observations of foundation temperature [22]. Constraining mixed layer salinity is limited to in situ observations. The introduction of remote sensing of sea surface salinity will enhance the observing system noting the expected limitations in accuracy of both Aquarius and SMOS (ESA's Soil Moisture and Ocean Salinity) missions. The depth of the mixed layer presently relies on an accurate background state as determined by the surface stress and buoyancy fluxes. The sparsity of in situ observations as well as the short decorrelation length scales limits the impact. Increasing the spatial and temporal density of in situ sampling would improve estimates of mixed layer depth and help identify systematic errors in the parameterisations and fluxes.

There are several second order problems that must also be addressed to enable the hydrodynamic models to correctly evolve the geostrophic turbulent interactions and add significant value over persistence skill. These include: (a) distributing the state anomalies in the vertical, (b) determine the secondary circulation properties of eddies and (c) initialise the fronts between mesoscale eddies. The present observing system does not adequately observe these properties and therefore places higher weighting on the background estimate. The influence of in situ profiles that observe eddies is constrained to the eddy scale and time decorrelation of vertical adjustment. Sampling all eddies would represent an order of magnitude increase in Argo which is expected to be infeasible and not a requirement of the climate community. An effective strategy would be to identify and profile the "large" eddies that have higher rotational inertia, stability and persistence. Two potential options for achieving this sampling are adaptive cycling from the Argo array and the implementation of a global array of gliders (or hybrid Argo/Glider design) that can be tasked for eddy profiling.
Observing the secondary rotation of eddies can be obtained from surface drifting buoys that are drogued within the stable surface circulation of the eddy. An example of the analysis of surface drifting buoy trajectory within an eddy is shown in Fig. $7 \mathrm{~g}$. There are several limitations to observing eddies however, the existing program could consider adapting the deployments to sample eddies. The introduction of wide-swath and constellation altimetry would improve the performance of the secondary circulation.

Ocean fronts have a lower dynamic height range compared to eddies, which makes them more difficult to observe with the observation errors of sea level anomaly products from current altimetry. Drifting buoys transiently observe the streamlines associated with fronts. However, the present coverage of drifting buoys will sample only a small fraction of the ocean fronts globally. Acoustic Doppler observations of the vertical current structure provide high quality information to constrain secondary circulation and fronts. Arrays of acoustic Dopplers would be required to observe the mesoscale. Remote sensing instrumentation that can observe the surface motion would also provide a very useful constraint. Glider and animal observer profiling has also been shown to be effective sampling the in situ properties of fronts.

Boundary currents, the Antarctic Circumpolar Current and throughflows provide specific challenges to Lagrangian based in situ observing systems. Novel approaches are required to adequately observe these important systems for global ocean circulation. Coordination of national coastal and shelf observing systems with international observing systems for HF radars, gliders, moorings, acoustic doppler, surface drifting buoys and remote sensing is required.

\section{STATUS OF OCEAN FORECASTING SYSTEMS}

Ocean prediction of the mesoscale ocean circulation is a grand challenge in oceanography. The Global Ocean Data Assimilation Experiment (GODAE) has provided an international focus over the past decade to accelerate progress. In 2008, a final symposium was held to draw to a close and review the achievements of GODAE over the previous 10 years. The achievements and status was documented in a series of community papers and subsequently published in a special issue of Oceanography. The specific advances in ocean prediction over the past decade were documented in [18]. At the conclusion of GODAE, there were 10 first or second generation operational ocean prediction systems from Australia, Asia, Europe and North America [3]. These systems consist of an ocean data assimilation system that assimilates altimetry, satellite SST and profile observations, and an eddy-resolving ocean general circulation model that is forced by surface 
fluxes from an operational numerical weather prediction system. The present systems share a common observation network but have unique assimilation methods, ocean models and surface flux products. A prediction system intercomparison was undertaken [21], which demonstrated that all systems are providing comparable nowcast performance, and forecasts that have measurable skill. For example, the performance of the BLUElink (analysis and forecast), HYCOMNCODA (HYbrid Coordinate Ocean Model-Navy Coupled Ocean Data Assimilation) (forecast), Mercator Ocean (analysis) and UK Met FOAM (Forecasting Ocean Atmospheric Model) (analysis) for the southeast Indian Ocean is summarised in a Taylor diagram [23] in
Fig. 8. The southeast Indian Ocean is a mid-latitude region that is characterised by geostrophic turbulence with an eastern boundary current. The anomaly correlation for the systems in this region range between 0.5 and 0.6 , which is at the threshold of what is generally regarded as skilful.

The performance of current forecasting systems is sufficient to impact some applications, [4 and 5] particularly if there is additional time to analyse the forecast in more detail or seek a consensus among the different forecast systems. Continued development of the ocean prediction system will be essential to improve the performance to have a greater impact on the widest range of applications.
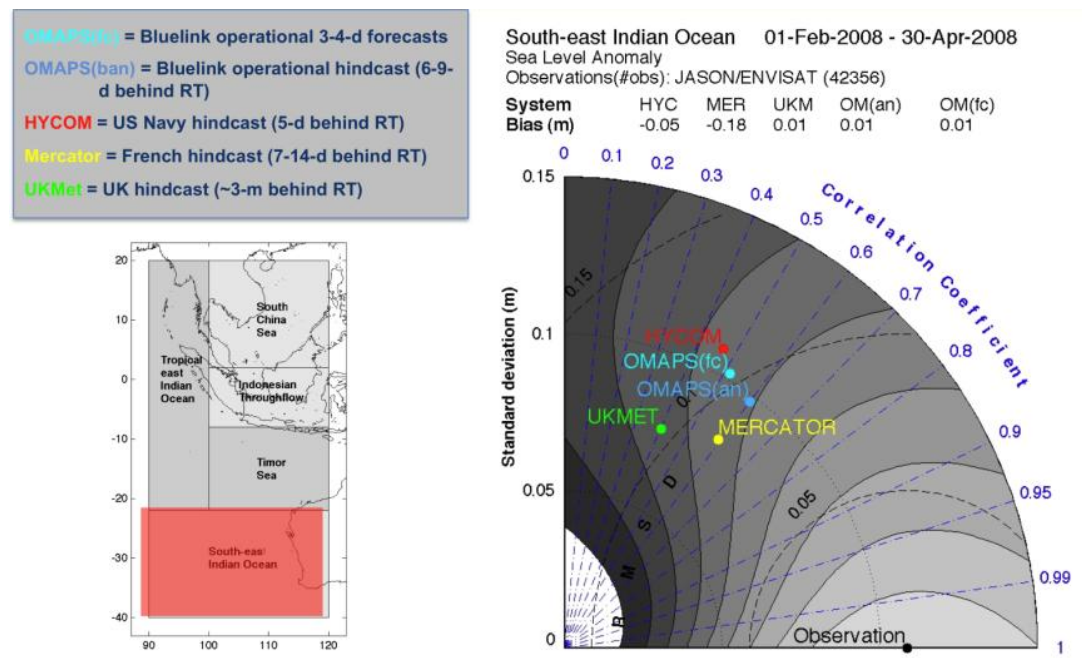

Figure 8: Example of the performance of GODAE global systems in the southeast Indian Ocean for sea level anomaly between $1 \mathrm{Feb}$ and $30 \mathrm{Apr}$ 2008. The anomaly correlation, root mean square error and model variability is summarised using a Taylor diagram [23]. The diagram is extended to include a shading for contours of equivalent skill for comparing models with different resolutions.

\section{OBSERVATIONAL REQUIREMENTS}

\subsection{Principles}

There are several fundamental principles that were adopted over the past decade that have been responsible for maximising innovation across a wide range of societal benefit areas including short-range ocean forecasting services. Retaining these principles over the next decade will be essential for the continued development of improved quality ocean forecasting services from existing systems and support capacity being developed in nations with maritime interests.

\section{One GOOS}

The ocean community must continue to work together on one GOOS to achieve the maximum societal benefit of information across all timescales, short-range, medium-range, intra-seasonal, seasonal and climate. OceanObs'09 wherever possible should encourage observing system campaigns that target one benefit area to consider other benefit areas in their design. For example, observational campaigns targeting ocean forecasting should seek to use high quality/standard instrumentation that can contribute to the climate record. Conversely observational campaigns targeting climate monitoring should seek to report in real-time to both maximise the observations retrieved but also make these available to ocean forecasting systems. In general achieving the extension in design across the benefit areas comes under pressure due the additional cost. The costs however must be set against the benefits the application already gains from other components of GOOS that do adhere to the same principles.

Open data access policy

Establishing a new paradigm in global data sharing was a major achievement of OceanObs'99 and critical to the development of capacity and maximising research 
innovation. Adherence to this policy in OceanObs'09 for new and emerging contributions from existing and new providers will be essential to retaining confidence from existing providers in the continued benefit ratios that were enjoyed over the past decade. The access to data has been underpinned by the coordination of a network of data assembly centres that have exploited a range of technologies enabling live access via the internet. Significant progress has also been undertaken to address the absence of community wide standards for data processing and metadata descriptions. Further progress to translate the community standards toward international standards will enable the ocean community to integrate data archives with other international communities and maximise the utilisation of open source software.

\section{Real-time monitoring}

Establishing the new paradigm of real-time monitoring for much of GOOS was another major achievement of OceanObs'99. This permits continuous monitoring of the observing system enabling actions to be taken to ensure the network continues to achieve its intended design and maximises the retrievals. Real-time observations have also been critical to providing a range of services through their synthesis for nowcasts and forecasts and their use in validation.

\subsection{New Principles - Operationalisation}

Many elements of GOOS have established themselves as having robust technologies delivering high quality observations that are critical to the continued monitoring of the ocean and climate. Real-time ocean analysis and forecast services similarly depend on the continued real-time monitoring of the ocean. The community must therefore engage in a debate on the pathway to sustain the observing system and operationalisation. The term "operational" is relatively new to oceanography and there is a significant apprehension within the research community to this phase of maturity. A simple definition of the difference between operational and research objectives is that the former maintains systems $(\mathrm{d} / \mathrm{dt}=0)$ while the latter seeks new challenges and innovation $(\mathrm{d} / \mathrm{dt} \neq 0)$. The drive and motivations to maintain and sustain are very different to the drive and motivations to innovate and change. There are several reasonable concerns within the research community on this transition and others that are less reasonable. A primary concern relates to the maintenance of quality and standards achieved under research institutes being maintained by agencies. There are concerns that mounting the case and identifying the sources of appropriated resources will take longer than the removal of research funds potentially creating a gap. There are also concerns relating to the sustainability of funding from appropriated resources once established. There are of course ways to resolve these concerns provided the community could agree to commit to such a pathway. For example, ensuring the quality and coverage is continually met might mean that $10 \%$ more observing platforms must be written into the operational design because of what has been learnt about the failure rates in research mode. Attracting the appropriated resources requires a robust and competitive benefit analysis. However, a one-off benefit analysis is not sufficient to sustain funding and the community must commit to reviewing benefit analyses to keep them current. A successful transition would therefore require that the research community remain engaged and provide on-going support to the operational communities that need to undergo training and develop a thorough understanding of the observing platform including the implications to services. Research communities however have few incentives to provide this support beyond the timeframe of the research funding with the attention and interest needing to be drawn toward the next research platform and next funding proposal. Operational funding proposals therefore need to include funding to contract the research community to provide the necessary support.

There are significant implications for the research community in not undergoing a transition to operations. For example, retention of funding for mature platforms of GOOS within research resources restricts the proportion of funding that can be directed toward the next upgrade of existing platforms as well as the funding for new technological innovations. Innovations in ocean observing technologies remain critical to improving the efficiency, coverage and quality of the observing system.

Sustaining what has been achieved by GOOS and making targeted extensions over the next decade will be critical to achieving robust delivery of consistently high quality ocean forecast services. Addressing the right research/operational composition to support GOOS over the next decade will be a continuing debate at the national and international level.

\subsection{OOFS Observing System Requirements}

The performance of operational ocean forecasting systems (now at or approaching second generation) is now able to produce a range of services that are beginning to be trialled, evaluated and applied by the user community such as the oil spill incident discussed in Sect. 3. The quality of the products requires an ocean forecaster trained to correctly interpret the information including evaluating the system diagnostics and developing an understanding of the biases. In applications where there is time to add value through error estimation and bias corrections the impact can be greater.

It is a simple statement to make that GOOS in 2009 under-samples the temporal and spatial scales of the 
mesoscale ocean. However, it is far more difficult to make a strong case for expanding GOOS when present ocean prediction systems do not yet efficiently use the information available [24]. The largest gains in performance over the next decade will be made by scientific and technical improvements to the prediction systems to approach more optimal performance rather than expansions to the observing system. Stronger evidenced based statements will emerge over the next 5 years on the specific observational requirements. We therefore recommend that sustaining or incrementally expanding GOOS will serve the majority of ocean prediction applications over this period. However, there are a number of applications such as SAR (Synthetic Aperture Radar) and MAES (Marine Accident Emergency Support) for which improvements in the observing system are more critical and would not be adequately served by waiting for the improvements to ocean forecast systems. Accurate nowcasts of the ocean conditions local to an incident has a high likelihood of positively impacting the outcome. In this case, we would encourage the observational community to consider approaches that could support this need. Specifically we advocate the implementation of adaptive sampling strategies outlined in Sect. 6.4.

Despite the sub-optimal performance of current ocean forecasting systems. we can none-the-less extrapolate the available evidence to describe the observational requirements for ocean nowcasting and forecasting systems in Tab. 6.1.

Translating these requirements into recommendations relative to specific observing platforms is the task of OceanObs'09 and the ocean observational community. However, based on the existing observing system we can comment on those platforms that have a significant impact and would be strongly encouraged to be given on-going support to sustain and extend over the next decade.

\section{Sea surface height}

- Support the on-going work of OSTST

- Altimetry coverage remains a limitation for realtime nowcasting

- Interleaved Jason-1, Jason2 and ENVISAT (Environmental Satellite) clear performance gain

- Strong support for Jason-3

- Support both the wide-swath and constellation proposals
- Require rapid development of real-time products from new satellite platforms

\begin{tabular}{|c|c|c|c|}
\hline Variable & Coverage & Resolution & Precision \\
\hline Sea level anomaly & $\begin{array}{l}\text { Global } \\
24 \mathrm{hr}\end{array}$ & $\begin{array}{l}<10 \mathrm{~km} \text { (open) } \\
<5 \mathrm{~km} \text { (coastal) }\end{array}$ & $<5 \mathrm{~cm}$ \\
\hline $\begin{array}{l}\text { Sea surface } \\
\text { temperature }\end{array}$ & $\begin{array}{l}\text { Global } \\
6 \mathrm{hr}\end{array}$ & $\begin{array}{l}<10 \mathrm{~km} \text { (open) } \\
<1 \mathrm{~km} \text { (coastal) }\end{array}$ & $<0.1 \mathrm{~K}$ \\
\hline $\begin{array}{ll}\text { Sea } & \text { surface } \\
\text { salinity } & \end{array}$ & $\begin{array}{l}\text { Global } \\
24 \mathrm{hr}\end{array}$ & $\begin{array}{l}<10 \mathrm{~km} \text { (open) } \\
<5 \mathrm{~km} \text { (coastal) }\end{array}$ & $<0.2 \mathrm{psu}$ \\
\hline $\begin{array}{l}\text { Sea surface } \\
\text { currents }\end{array}$ & $\begin{array}{l}\text { Global } \\
24 \mathrm{hr}\end{array}$ & $\begin{array}{l}<10 \mathrm{~km} \text { (open) } \\
<5 \mathrm{~km} \text { (coastal) }\end{array}$ & $<0.1 \mathrm{~ms}^{-1}$ \\
\hline $\begin{array}{l}\text { Sub-surface } \\
\text { temperature, } \\
\text { salinity, pressure } \\
(0-200 \mathrm{~m})\end{array}$ & $\begin{array}{l}\text { Global } \\
24 \mathrm{hr}\end{array}$ & $\begin{array}{l}<100 \mathrm{~km} \text { (open) } \\
<50 \mathrm{~km} \text { (coastal) }\end{array}$ & $\begin{array}{l}<0.5 \mathrm{~K} \\
<0.5 \mathrm{psu}\end{array}$ \\
\hline $\begin{array}{l}\text { Sub-surface } \\
\text { currents }\end{array}$ & $\begin{array}{l}\text { Global } \\
24 \mathrm{hr}\end{array}$ & $\begin{array}{l}<100 \mathrm{~km} \text { (open) } \\
<50 \mathrm{~km} \text { (coastal) }\end{array}$ & $<0.1 \mathrm{~ms}^{-1}$ \\
\hline Sea ice coverage & $\begin{array}{l}\text { Global } \\
6 \mathrm{hr}\end{array}$ & $<10 \mathrm{~km}$ & $<25 \%$ \\
\hline Sea-ice thickness & $\begin{array}{l}\text { Global } \\
24 \mathrm{hr}\end{array}$ & $<10 \mathrm{~km}$ & $<0.1 \mathrm{~m}$ \\
\hline River discharge & $\begin{array}{l}\text { Global } \\
1 \mathrm{hr}\end{array}$ & $<1 \mathrm{~km}$ & $<1 \mathrm{~cm}$ \\
\hline Tides & $\begin{array}{l}\text { Global } \\
1 \mathrm{hr}\end{array}$ & $<1 \mathrm{~km}$ & $<1 \mathrm{~cm}$ \\
\hline Momentum flux & $\begin{array}{l}\text { Global } \\
1 \mathrm{hr}\end{array}$ & $<10 \mathrm{~km}$ & $<0.01 \mathrm{Nm}^{-2}$ \\
\hline Heat flux & $\begin{array}{l}\text { Global } \\
1 \mathrm{hr}\end{array}$ & $<10 \mathrm{~km}$ & $<10 \mathrm{Wm}^{-2}$ \\
\hline Mass flux & $\begin{array}{l}\text { Global } \\
1 \mathrm{hr}\end{array}$ & $<10 \mathrm{~km}$ & $<1 \mathrm{~mm} / \mathrm{hr}$ \\
\hline
\end{tabular}

Table 6.1: Observational requirements for operational ocean forecast systems [25]

Sea surface temperature

- Support the on-going work of GHRSST (Global High Resolution Sea Surface Temperature)

- Support the maintenance of the multi-sensor, multiorbit SST network

- Support the continuance of microwave and geostationary SST (Sea Surface Temperature)

Sea surface salinity

- Support the research and development of remote sensing missions

- Proposed SSS (Sea Surface Salinity) accuracy is a limit to impact in OOFS (Operational Ocean Forecasting Systems) 


\section{$\underline{\text { Sea surface currents }}$}

- Global remote sensing of ocean currents is specific observing gap

- Many applications are sensitive to the large errors

- Encourage progress in this area

- Encourage an increased density of drifting buoys sufficient to have a measurable impact on the nowcast/forecast of surface currents

Sub-surface T,S,P

- In situ will likely always undersample the mesoscale (no redundancy)

- 10 times Argo-2009 would still undersample the mesoscale

- Support sustaining and incrementally increasing Argo

- Double cycling to 5 days for Iridium communications

- Support maintenance of XBT's

The design of the analysis cycle and the performance of operational ocean forecasting systems are critically dependent on the properties of each component of the observing system in terms of, timeliness, quality, homogeneity and robustness. The specific requirements for each property are summarised in Tab. 6.2.

\begin{tabular}{|l|l|}
\hline Property & Requirements \\
\hline Timeliness & $\begin{array}{l}\text { OOFS target analysis within 24 hrs of real- } \\
\text { time } \\
\text { Real-time reporting within 12 hrs of real- } \\
\text { time } \\
\text { Global coverage within 36 hrs of real-time }\end{array}$ \\
\hline $\begin{array}{l}\text { Automatic } \\
\text { quality control }\end{array}$ & $\begin{array}{l}\text { Removal of all bad observations } \\
\text { Synchronise automatic and delayed mode } \\
\text { QC procedures, Analyse and intercompare } \\
\text { QC systems }\end{array}$ \\
\hline Homogeneity & $\begin{array}{l}\text { Homogeneous sampling in space, time and } \\
\text { errors, Target 24 hr uniform spatial } \\
\text { coverage }\end{array}$ \\
\hline Robust & $\begin{array}{l}\text { Maximise platform availability of } \\
\text { observing system, Build redundancy to } \\
\text { maintain minimum design } \\
\text { Operationalise components of the } \\
\text { observing system }\end{array}$ \\
\hline
\end{tabular}

Table 6.2: Essential properties and requirements for operational ocean forecast systems
The delay in full orbit coverage from Jason 1 and Jason 2 as well as the 3-day delay in the delivery of IGDR products influences the timeliness of the analysis. For example the OceanMAPS system [10] performs a +/- 5 day symmetric analysis 9 days behind real-time and a +2 day / 5 day asymmetric near real-time analysis 5 days behind real-time. The improvements to the analysis design and system performance from each of the properties outlined in Tab. 6.2 is shown schematically in Fig. 9.

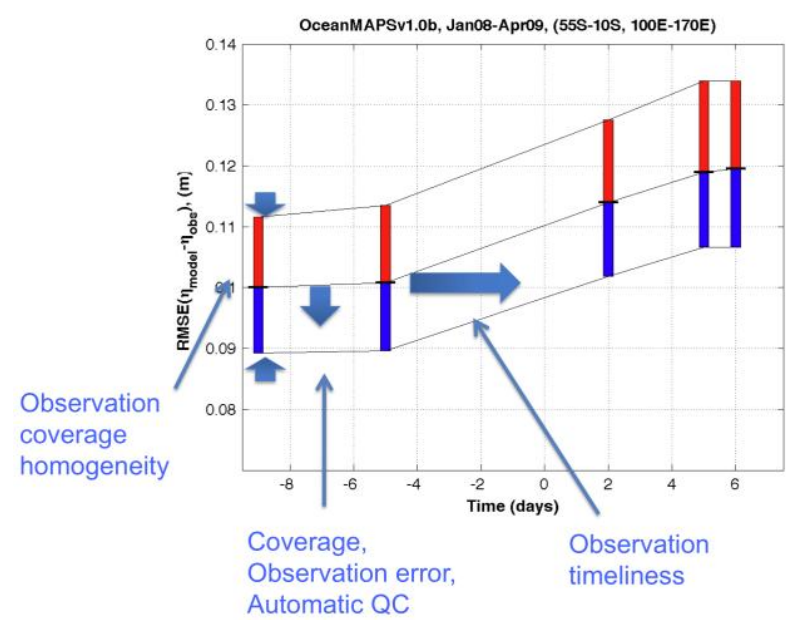

Figure 9: The distribution of root mean square error of sea level anomaly for all forecast cycles of OceanMAPSv1.0b from Jan 2008 to Apr 2009. Overlaid is a schematic of the impact of improvements from the properties in Tab. 5.2.

\subsection{Adaptive Observational Sampling}

The applications identified in Tab. 1.1 in general require high quality nowcast and forecast information that is time critical. The observational requirements for these applications will not be satisfied by GOOS in 2009 or from incremental improvements. In the majority of cases, these applications occur at ad hoc times and ad hoc locations, which cannot be predicted with sufficient precision to design a fixed observing system. In this case, the observing system requires some capacity to, in a short activation time, increase the sampling within the region of the incident, so-called adaptive sampling. 
6.5. There are several in situ platforms that could be considered to develop a capacity to adaptively observe the ocean:

a) Gliders - provide rapid sampling and programmable and adaptive pathways. However, current designs offer limited ranges and longevity due to battery life. Gliders can also experience difficulty in strong currents. Adaptive sampling would require the capacity for aerial deployments to the target regions and offshore retrieval.

b) AUV - provide rapid sampling, have a capacity for an extensive range of sensor equipment, are programmable for adaptive pathways and more control over the speed and operating conditions compared with gliders. In general, these instruments are more expensive and less expendable.

c) Argo-Glider hybrid - An advantage of Argo is that it is distributed globally such that an Argo is always near to an event. Some glider capability in the design of an Argo would permit tasking to move the float closer to the incident and perform adaptive profiling. A global array of Argo-gliders could cycle as an Argo float for the majority of its lifetime contributing to the climate record and then be tasked on an event basis.

d) Argo adaptive cycling - Iridium permits two-way communications which could be used to change cycling patterns. Argo could be tasked for specific incidents to cycle more frequently and over a different depth range. Post incident the Argo float could resume normal operations.

e) Drifting buoys - SVP buoys are a compact instrument that is packaged for easy deployment. A fleet of SVP buoys could be deployed for rapid assessment of the ocean circulation. Aerial deployment packages are also available.

Developing this capacity will require a coordinated international and national effort to plan, design, evaluate and implement a system of systems. A similar international charter for space and major disasters (http://www.disasterscharter.org) has been developed amongst the world's space agencies to respond to major disasters with relevant satellite products including the tasking of active sensors. No equivalent international charter exists for the in situ community, largely because the data products already have an open access data policy. Development of the capacity to deploy adaptive in situ observations would require an international framework and an equivalent charter to manage activations.

\subsection{Justification}

The observational requirements for operational ocean forecasting systems over the next decade are significant and will require the robust evidence on the societal benefits to make the case and justify the sustained resources. At the simplest level, the case that needs to be made is an economic question of the cost versus benefit. The relationship of this to the observing system requirements requires that we define a series of secondary relationships as shown in Fig. 10. The first step is to translate user requirements into a performance vs benefit curve i.e., the performance of the nowcast/forecast system versus the benefit to the users' application. Benefit can be defined in economic terms, societal impacts etc. The required performance might be achieved by improvements to the system, which will result in higher costs for research and development and resources to operate the system. Alternatively, performance can be improved through more stringent observational requirements, which will also result in a greater cost. The total cost to achieve a given improved benefit would therefore be a trade-off between the system improvements and additional observational requirements. In Fig. 10, the blue circles represent information that can be obtained from the operational

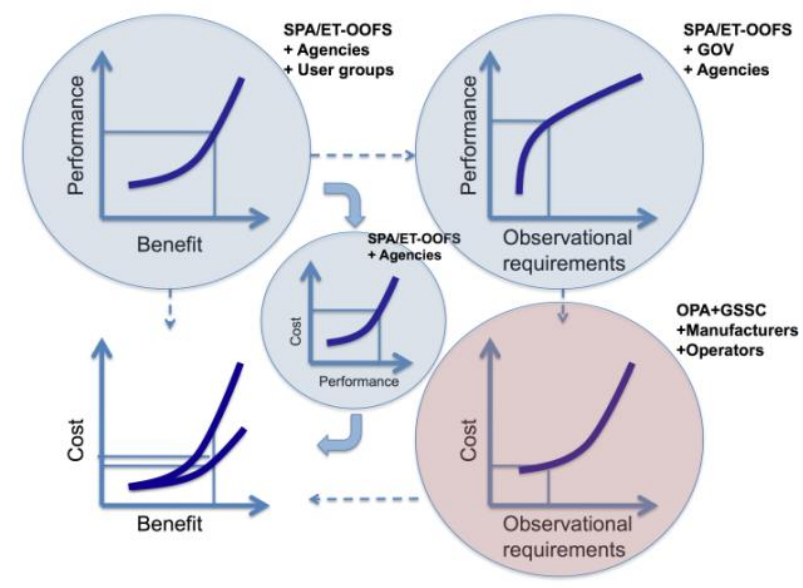

Figure 10: A schematic diagram of the cost-benefit analysis of ocean prediction and the relationship to ocean observing systems. The benefit is assumed to be related to the performance of the services provided by national agencies and institutions. The performance is assumed to have a functional relationship to the observational requirements, which ultimately comes at a cost.

ocean forecasting community, while the pink circle represents information that can be obtained from the ocean observing community.

Obtaining substantial evidence of the impacts or benefits of operational ocean forecast products on the wide range of potential applications has been slow to develop. The performance of first generation forecasting systems is a limiting factor. At present user champions are rare and need to be supported and encouraged to provide the feedback on the products and the user requirements. Support could take the form of training or sponsorship to attend meetings or other practical incentives. Users are typically operators who have day 
jobs and limited time to perform work that may or may not yield an immediate impact to their applications. Agencies and other institutions will need to meet users beyond halfway with the current product suite. Improved performance over the next 5 years will provide more obvious impacts. Once operators gain confidence in the information to make decisions, advocacy will become easier to obtain and partnerships will strengthen. JCOMM ET-OOFS will seek to coordinate the many national efforts to engage users into a consolidated international context.

The performance versus observational requirements can be informed by observing system evaluations (e.g., [26]), observing system design analysis, as well as new advances in technologies or utilising new platforms. A task team has been proposed under GODAE OceanView to develop the capability to respond to these questions. The costs for various observational designs can be specified by the ocean observing community. Once the required information is obtained, finding the optimum benefit for a given cost becomes an operations research problem.

\section{CONCLUSIONS}

Over the past decade, several agencies and institutions have developed the capability for global and regional operational ocean prediction. These systems have demonstrated that the observations available from the global ocean observing system in 2009 are sufficient to constrain mesoscale ocean variability to produce skilful forecasts. The performance of these systems is modest and is only able to impact a small fraction of the potential applications. Due to the relative maturity and computational expense of global ocean forecasting, current systems have adopted less optimal configurations. The dominant source of improvement over the next 5 years is expected to come from optimisation of the data assimilation, initialisation, models and surface fluxes. None the less GOOS currently under-samples the mesoscale ocean variability and there is no redundancy in the observing system for ocean forecasting. The highest priority to the ocean forecasting community would be to consolidate and incrementally extend the existing system and to encourage the transition of mature observing platforms to operational systems. Within the next 10 years, as systems approach more optimal performance, more detailed and quantitative evidence will help to define more rigorously the observational requirements. The requirements for high impact applications are expected to be beyond the capability of the GOOS $+10 \%$ and instead require new strategies to adaptively enhance the observing systems and reach the threshold in forecast skill. Developing this capability and establishing the capacity throughout the ocean basins will pose a significant challenge to the observational community.
The ocean forecasting community will have a role to play in developing the cost versus benefit analysis to justify the implementation at the national level with international coordination.

Over the next decade, a grand challenge for ocean prediction will be to integrate with numerical weather prediction as coupled systems for short- to mediumrange prediction. A minimum criterion will be for ocean prediction systems to produce a skilful SST forecast. The maintenance of a high quality and homogeneous global ocean observing system will be critical as well as targeted observing campaigns to tune and calibrate boundary layer and air-sea flux parameterisations.

\section{ACKNOWLEDGEMENTS}

We gratefully acknowledge contributions to this paper from Leon Majewski, the NASA Ocean Biology Processing Ocean Variability evaluated from an Ensemble of Ocean Synthesis Group, the Australian Bureau of Meteorology, Nicolas Summons, and Dr. Brian King, APASA.

\section{REFERENCES}

1. Smith, N. and M. LeFebvre, 1997: The Global Ocean Data Assimilation Experiment (GODAE). Monitoring the oceans in the 2000s: An integrated approach, International Symposium, Biarritz, 15-17 October.

2. Le Traon, P. Y., and co-authors, 2001: Operational oceanography and prediction: A GODAE perspective, in Koblinsky, C. J. and N. R. Smith (eds), Observing the oceans in the 21 st century, Bureau of Meteorology, Melbourne.

3. Dombrowsky, E., and co-authors, 2009: GODAE systems in operation, Oceanography, 22(3), 76-91

4. Davidson, F., and co-authors, 2009: Application of GODAE ocean current forecasts to search and rescue and ship routing, Oceanography, 22(3), 172-177

5. Jacobs, G. A., R. Woodham, D. Jourdan and J. Braithwaite, 2009: GODAE applications useful to Navies throughout the world, Oceanography, 22(3), 182-189

6. Goni et al., 2009: Applications of satellite-derived ocean measurements to tropical cyclone intensity forecasting, Oceanography, 22(3),

7. Hackett, B., E. Comerma, P. Daniel and H. Ichikawa, 2009: Marine oil pollution prediction, Oceanography, 22(3), 168-175

8. Brassington, G. B., 2009: Ocean prediction issues related to weather and climate prediction, CAS XV Vision paper (Agenda item 8.5)

9. Seaman, R., and co-authors, 1995: Evolution of the Bureau of Meteorology's Global Assimilation and Prediction System, Part 1: Analyses and Initialization, Aust. Met. Mag., 44, 1-18 
10. Brassington, G. B., and co-authors, 2007: BLUElink> Development of operational oceanography and servicing in Australia, Journal of Research and Practice in Information Technology, 39, 151-164

11. Barron C. N., and co-authors, 2003: Global ocean nowcasts and forecasts with the Navy Coastal Ocean Model (NCOM), NRL Review ocean science and technology, 175-178

12. Deng, X, DA Griffin, K. Ridgway, J.A. Church, W.E. Featherstone, N. White and M. Cahill (2009). Satellite altimetry for geodetic, oceanographic and climate studies in the Australian region, in: Vignudelli S.K.A. and Cipollini P. (eds.), Coastal Altimetry, Springer, Berlin

13. Chelton, D. B., M. G. Schlax, R. M. Samelson, and R. A. de Szoeke, 2007: Global observations of large oceanic eddies. Geophys. Res. Lett., 34, L15606, doi:10.1029/2007GL030812

14. Maximenko, N. A., O. V. Melnichenko, P.P. Niiler, and H. Sasaki, 2008: Stationary mesoscale jet-like features in the ocean, Geophys. Res. Lett., 35, L08603, doi:10/1029/2008GL033267.

15. Brassington, G. B., 2009: Estimating surface divergence of ocean eddies using observed trajectories from a surface drifting buoy, J. Atmos. and Oceanic Tech., doi:10.1175/2009JTECHO651.1

16. Griffies, S. \& Co-Authors (2010). "Problems and Prospects in Large-Scale Ocean Circulation Models" in these proceedings (Vol. 2), doi:10.5270/OceanObs09.cwp.38

17. Hurlburt, H.E., and P.J. Hogan. 2000: Impact of $1 / 8^{\circ}$ to $1 / 64^{\circ}$ resolution on Gulf Stream model-data comparisons in basin-scale subtropical Atlantic Ocean models. Dynamics of Atmospheres and Oceans 32:283329

18. Hurlburt, H. E., and co-authors, 2009: High resolution global and basin-scale ocean analyses and forecasts, Oceanography, 22(3), 80-97

19. Proceedings of the ECMWF workshop on OceanAtmosphere interactions, 10-12 November 2008, ECMWF, Reading, UK, Feb 2009

(http://www.ecmwf.int/publications/library/do/reference s/list/28022009)

20. Cummings, J., and co-authors, 2009: Ocean data assimilation systems for GODAE, Oceanography, 22(3), 96-109

21. Hernandez, F., and co-authors, 2009: Validation and intercomparison studies within GODAE, Oceanography, 22(3), 124-139

22. Donlon, C. J., and co-authors, 2002: Towards improved validation of satellite sea surface skin temperature measurements for Climate Research, J. Climate, 15, No. 4, 353-369.

23. Taylor, K. E., 2001: Summarizing multiple aspects of model performance in a single diagram, JGR, 106, D7, 7183-7192
24. Oke, P. R. and A. Schiller, 2007: Impact of Argo, SST and altimeter data on an eddy-resolving ocean reanalysis, Geophysical research letters, 34, L19601, doi:10.1029/2007/GL031549

25. JCOMM Service Program Area, statement of guidance, 2008, JCOMM MR-53

26. Oke P. R., and co-authors, 2009: Observing system evaluations using GODAE systems, Oceanography, 22(3), 144-153 